\title{
Abstractions
}

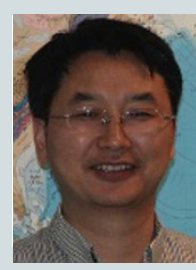

\section{FIRST AUTHOR}

Predicting earthquakes has proved an elusive goal for seismologists, in part because direct measurements from deep inside faults have been lacking. California's

San Andreas Fault Observatory at Depth (SAFOD) site now allows scientists deep access to the world's most-instrumented section of fault. There, Fenglin Niu of Rice University in Houston, Texas, and his colleagues fired experimental pressuregenerated pulses and monitored the time taken for the resulting seismic waves to travel through a 10-metre section of rock at a depth of 1 kilometre. On page 204, they confirm that stress-induced changes occur at the San Andreas Fault in California shortly before rupture events. Niu tells Nature that if such changes also occur elsewhere, reliable earthquake prediction may be possible.

What efforts have been made in the past to predict earthquakes?

Laboratory studies in the 1960 s showed that the speed of seismic waves travelling through a rock varies with the level of stress applied to the rock owing to the opening and closing of micro-cracks. And that microcrack density increases rapidly before an earthquake. But failure to replicate those findings in the field dampened interest.

What work has been done since then? Researchers have been trying to precisely measure changes in the velocity of seismic waves at fault depth. Recent technological improvements, including repeatable pulse sources, high sampling rate data-acquisition systems and massive data-processing capability, have made measurements reliable. At SAFOD, earthquake locations can be very accurately determined, making reliable estimates of changes in stress possible.

Were earthquake events a help or a hindrance to your research?

They provided valuable data. We wanted to show that stress-dependent seismic velocity seen at the surface also occurs deep within faults. There were two anomalous spikes in our data. Co-author Tom Daley wondered whether the spikes could be signals of changes in tectonic stress. Our calculations show that the spikes occurred 10 hours before a magnitude- 3 earthquake and 2 hours before a magnitude-1 earthquake, which suggests that they relate to pre-rupture stress.

Do you think earthquake prediction will become possible during your career? These initial data are encouraging, but are just the start. We need to establish whether these signals are pervasive at different sites, and, if so, how the signal timing relates to earthquake size.
MAKING THE PAPER

\section{Erica Ollmann Saphire}

\section{Structure reveals Ebola virus strengths and weaknesses.}

Erica Ollmann Saphire staked her young lab's future on solving the crystal structure of the glycoprotein on the surface of Ebola virus. The move was risky, because the protein is notoriously difficult to express and crystallize in a stable and ordered form. And at least three other groups were racing her for the same solution. Although the high-stakes competition made her "really nervous", Ollmann Saphire, an immunologist at the Scripps Research Institute in La Jolla, California, and her team won the race and provide a picture of how the deadly virus evades immunity and enters cells.

The work was long and difficult. Over four years, the group expressed 130 versions of the Ebola virus glycoprotein, grew 50,000 crystals, made 30 trips to two synchrotrons to bounce $\mathrm{X}$-rays off the crystal, and tested the diffraction of more than 800 crystals.

What the team found may explain how the virus can lurk for years and still prove so virulent. The structure reveals a 'candy-floss' coating of disordered protein and carbohydrate. The coating keeps the virus stable in the environment and hides the glycoprotein's binding mechanism from attacking host immune cells.

The findings suggest that the glycoprotein acts like a spearfishing line: three attachment subunits are tied together by three fusion subunits, which are wound around them like the line on a spool. Once a possible host contacts the virus, a protease snips the cloak away. Then the attachment subunits are released, triggering the fusion subunits to spear the human cell, allowing infection. "This virus is a fascinating little beast," says Ollmann Saphire. "It's a stripped-down, tiny machine of just seven genes, with all sorts of structural tricks

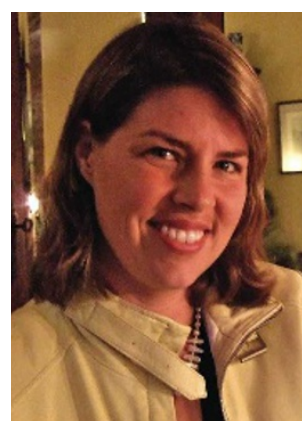

to replicate itself while evading the immune system."

The team also managed to solve the structure of an infection-blocking antibody bound to the viral protein. This revealed a vulnerability in the virus - an exposed site on the glycoprotein. The antibody evidently bridged two glycoprotein subunits, somehow preventing it from 'spearing' a human cell. Getting a picture of this site, and a few other sites where antibodies might bind to the glycoprotein, could provide a route to a therapeutic defence against the virus.

Getting that picture proved a "major slog", says Ollmann Saphire. Of the 50,000 crystals the team grew of the glycoprotein-antibody complex, only one diffracted the $\mathrm{X}$-rays to high enough resolution to close in on the structure. And after identifying this there were still obstacles to overcome, because phase information is not collected during X-ray diffraction work, but is required to assemble the data into a three-dimensional model.

The standard structural biology solution to this problem is either to use a similar, already solved structure or to add a heavy metal to your protein. Neither approach worked here. Instead, with the help of lab tech Ann Hessell, the researchers tried replacing the methionine amino acids in the antibody with seleniumcontaining methionines. Surprisingly, they got higher-resolution data. "We don't know why the selenomethionine antibody worked," Ollmann Saphire says. "It just did."

Despite worries about putting all her eggs into one difficult-to-decipher basket, Ollmann Saphire says she was driven more by scientific curiosity than by career progression. "We didn't do this to get some big, shiny prize," she says. "This virus is just truly fascinating, and the structure was a problem that needed to be solved."

\section{FROM THE BLOGOSPHERE}

With the provocative title

"The end of theory", an essay in Wired magazine on 23 June argues that, with the advent of huge datasets and Googlelike algorithms, the scientific method has become obsolete. Inevitably, this view has stimulated responses in the scientific blogosphere, not least on Nature Network, where David Basanta of the University of Dundee, UK, points out in his blog (http:// tinyurl.com/4vc8pz) that models are "more than predictive machines" and that science involves true understanding, not just prediction.

Another Nature Network blogger, Bob O'Hara of the University of Helsinki (http://tinyurl.com/4tym73), says that "10 million observations on 6 samples is still only information about 6 samples." The quality of data is important, as well as the quantity.

Basanta's question "Does any one else think that traditional science is a thing of the past and that cloud computing will drive us modelers to the employment office?" is answered by Nature Network users with a resounding "no". 